\title{
Philosophiques
}

\section{L’esthétique de Rodolphe de Repentigny et la phénoménologie}

\section{Raymond Montpetit}

Volume 5, numéro 2, octobre 1978

URI : https://id.erudit.org/iderudit/203097ar

DOI : https://doi.org/10.7202/203097ar

Aller au sommaire du numéro

Éditeur(s)

Société de philosophie du Québec

ISSN

0316-2923 (imprimé)

1492-1391 (numérique)

Découvrir la revue

Citer cet article

Montpetit, R. (1978). L'esthétique de Rodolphe de Repentigny et la phénoménologie. Philosophiques, 5(2), 211-228.

https://doi.org/10.7202/203097ar d'utilisation que vous pouvez consulter en ligne.

https://apropos.erudit.org/fr/usagers/politique-dutilisation/ 


\title{
L'ESTHÉTIQUE DE RODOLPHE DE REPENTIGNY ET LA PHÉNOMÉNOLOGIE.
}

\author{
par Raymond Montpetit
}

«Le philosophe cesse d'écrire ou meurt, non parce qu'il a achevé son œuvre mais parce que, au-dessous de son projet total de vivre et de penser, quelque chose inopinément se défalque ... toute mort est prématurée au regard de la conscience qu'elle atteint, la vie et l'œuvre entière ne prennent finalement un sens irrévocable qu'aux yeux des survivants, et par l'illusion du spectateur étranger. »

Maurice Merleau-Ponty.

Cette remarque que Merleau-Ponty fait, au sujet de l'œuvre écrite du philosophe, (ici Descartes), s'applique à la lettre à l'activité du critique d'art Rodolphe de Repentigny. S'il est bien vrai qu'une æuvre, quelle qu'elle soit, n'est jamais achevée ou close, n'est jamais une totalité cohérente dont la signification serait une fois pour toutes fixe et prononcée, cela est d'autant plus vrai lorsqu'on est en face d'un ensemble de textes comme ceux qu'a écrits Repentigny, textes disparates, brefs, publiés sous forme de chroniques journalistiques, et dont la fin abrupte ne fût certes ni voulue, ni préméditée, ni d'aucune façon inscrite dans le projet d'écriture lui-même.

Oeuvre éminemment fragmentaire donc, dans sa production, mais aussi dans sa réception, si l'on tient par surcroît compte du fait que nous ne disposons d'aucune forme d'anthologie qui nous permettrait d'avoir un regard englobant sur la totalité de cette production. 
Devant ces textes, c'est de prime abord leur texture même qui nous frappe: on ne peut pas traiter les textes de Repentigny comme s'ils formaient un tout continu, un livre qui irait sans interruption, d'une introduction à une conclusion. La première conséquence de cette pluralité d'articles est qu'il est impossible d'y retrouver une conceptologie permanente transtextuelle, qui conserverait aux mots un sens fixe et précis ; tout comme dans l'esthétique de Repentigny, peignant sous le nom de Jauran, chaque tableau doit posséder sa propre logique interne dans une stratégie picturale qui est son style, chaque texte spécifique de Repentigny cherche à convaincre, à éduquer le lecteur sur un point, et les concepts utilisés se définissent alors souvent l'un l'autre à l'intérieur du réseau d'oppositions et d'équivalences propre à la démonstration particulière recherchée par le critique. Un exemple clair de cette structure conceptuelle stratégique et ponctuelle, qui s'oppose à une conceptologie généralisée qui se maintient unique dans tous les recoins de la théorie, serait celui du rôle d'une notion comme celle de "sensibilité " dans la production picturale. Tout dépend du texte où cette notion joue: lorsqu'elle s'oppose à celle d'objet pictural autonome, de tableau en-soi, elle devient un concept négatif. Par exemple, on apprend, en novembre 1955, que Jean-Paul Jérôme " ne fait pas étalage de sentimentalité ou de sensiblerie " ${ }^{1}$, et, en mai 1955, que l'énoncé direct d'un sentiment, d'une révolte, d'une attitude est de moins en moins recherché ${ }^{2}$. Mais, lorsque cette même notion de sensibilité est opposée à la pure technique et au procédé, alors elle devient un concept positif, et on lit que certains peintres ont le défaut " de n'être jamais parvenus à mettre la technique au service de la vie et de la sensibilité ${ }^{3}$.

Il en va de même de presque tous les concepts fondamentaux qu'utilise Repentigny: leur sens est structural et stratégique, il est indissociable du texte particulier à l'intérieur duquel il fonctionne dans un but précis.

1. La Presse, "Vigoureuse élimination de la sensiblerie dans la peinture de Jérôme ", 14 novembre 1955.

2. La Presse, "En atrendanr des murs à peindre ", 11 mai 1955

3. La Presse, "Exposition qui fera époque ", 2 mai 1953. 
Notre lecture nécessairement "lacunaire " et, pour ainsi dire, "poreuse » est donc la reprise en quelque sorte de cette pratique textuelle, elle-même intermittente et même interrompue.

Comment, malgré tout peut-on recevoir aujourd'hui ces textes, publiés par Repentigny principalement dans La Presse, après son retour d'Europe, soit de 1952 jusqu'à son décès, en juillet 1959 ? Vingt ans après, ils sont comme les symptômes de cette période, étant les marques des combats théoriques et sociaux que la peinture d'alors se devait de livrer.

Examinons un peu cette écriture, pour tenter d'en saisir les axes problématiques, la logique interne et les "a priori" philosophiques sur lesquels elle repose.

Comment situer la pratique textuelle de Repentigny, à l'intérieur d'une méthodologie générale de la critique et de l'interprétation des productions artistiques? Quels problèmes $\mathrm{y}$ sont thématisés et lesquels seraient, pour ainsi dire, occultés?

En examinant l'armature conceptuelle de la critique d'art, qui essentiellement provient elle-même de la critique littéraire, le statut de la peinture étant tributaire théoriquement de celui accordé à la littérature, Michel Foucault fait cette remarque des plus pertinentes :

"On pourrait envisager la manière dont la critique et $l^{\prime h i s t o i r e ~ l i t t e ́ r a i r e s, ~ a u x ~} 18^{\mathrm{e}}$ et $19^{\mathrm{e}}$ siècles, ont constitué le personnage de l'auteur et la figure de l'œuvre, en modifiant et déplaçant les procédés de l'exégèse religieuse et de la critique biblique ${ }^{4}$. 》

En effet, les concepts fondamentaux qui articulent la réflexion sur l'art sont souvent à base théologique et importés, sans critique, du contexte herméneutique, c'est-à-dire de cette discipline constituée pour interpréter le sens des textes bibliques et évangéliques où quelqu'un témoigne du sacré et interpelle ses semblables pour en faire des croyants.

Une autre source qui influe sur les discours d'art, il faut l'avouer, est celle de la « conversation mondaine ». Voici, par

4. Foucault, Michel, Lordre du discours, Paris, nrf/Gallimard, 1971, p. 66-67. 
exemple, ce que R. Jakobson affirmait en 1921 :

«Il n'y a pas longtemps encore, l'histoire de l'art .. n'était pas une science, mais une «causerie». Elle suivait toutes les lois de la causerie. Elle passait allégrement d'un thème à l'autre. La causerie ne connaît pas de terminologie précise : au contraire, la variété des termes, les mots équivoques... sont des qualités qui apportent du charme à la conversation. Ainsi l'histoire de l'art ne connaissait pas de terminologie scientifique, elle utilisait les mots du langage courant sans les faire passer au crible de la critique, sans les limiter avec précision"."

Qu'en est-il alors de la critique de Repentigny, par rapport à ces deux constatations, celle d'une conceptologie herméneutique et théologique, et celle d'une conversation purement mondaine?

Si la terminologie employée par Repentigny n'est pas, comme je le notais, fixe et définie, d'entrée de jeu il faut dire qu'elle n'est pas de l'ordre de la «causerie mondaine» dénoncée par Jakobson. La conception de la critique d'art et de la pratique picturale, qui s'y énonce, est celle d'une aventure existentielle où le peintre et sa collectivité se trouvent impliqués. La pratique artistique est donc prise au sérieux par Repentigny, et toute forme de "snobisme » en cette matière est par lui dénoncée et disqualifiée.

Les relations de cette critique d'art avec une conception herméneutique de "l'expression » sont plus complexes, mais, dans l'ensemble, je dirais que la piatique critique de Repentigny s'éloigne d'une conception herméneutique traditionnelle pour aller vers une gestion davantage phéno-structurale du pictural : je voudrais énumérer les principes fondamentaux de ce que j’appelle une approche herméneutique du phénomène artistique, pour voir ensuite, au fur et à mesure, si la critique de Repentigny s'y réfere ou non.

Premièrement, l'herméneutique, comme théorie, comme science de l'interprétation, croit que tous les media d'expression sont équivalents, c'est-à-dire qu'ils sont tous des expres-

5. JAkOHSON, Roman, "Du réalisme artistique ", in : Théorie de la littérature, en coll. Seuil, Paris, 1965, p. 98. 
sions de la nature humaine: il n'y a pas de coupure entre parole, monument, toile ou texte, tout cela étant susceptible d'un même mode de compréhension et d'un même traitement.

Ce principe n'est pas partagé par Repentigny, qui affirme au contraire la spécificité de la peinture en regard, par exemple, de l'expression verbale : ainsi, il affirme que ce qu'un peintre pense de l'art, il le dit par ses tableaux uniquement, non par des enquêtes verbales :

«Ce genre d'enquêtes adressées à des peintres dépasse de loin son but, quand on prétend en tirer des conclusions générales. Tout au plus pourrait-on en déduire comment les peintres s'expriment verbalement ou littérairement, il est malhonnête de prétendre en conclure ce qu'ils pensent de l'art. Cela, ils le disent dans leurs tableaux. ${ }^{6}$."

Ce qui se dit picturalement ne devait donc pas pouvoir se dire autrement : Repentigny est très clair là-dessus, affirmant, en novembre 1954 :

"La peinture est un langage direct, une action, et non pas, ce que l'on suppose généralement qu'est le langage parlé : une simple traduction de la pensée et de la perception ${ }^{7}$. "

Que le langage n'est pas la traduction d'une pensée qui le précéderait, c'est là une des thèses fondamentales de la phénoménologie du langage telle que la définit Maurice Merleau-Ponty, lorsqu'il disait au Colloque International de Phénoménologie en 1951 :

«Mes paroles me surprennent moi-même et m'enseignent ma pensée. »

Cette idée d'un art-action, comme le dit Repentigny, est aussi, selon Picon, la définition même d'une conscience moderne de l'art. Picon écrit :

«Il y a une conscience moderne de l'art qui, confrontée à la conscience qui la précède nous suggère qu'un art de création vient d'être substitué à un art d'expression. Avant l'art moderne, l'ouvre semble l'expression d'une expérience antérieure, l'œuvre dit ce qui a été conçu et vu. Pour l'art

6. La Presse, "Des peintres sunt trahis ", 26 janvier 1957.

7. La Presse, "Quelques leçons de Matisse ", novembre 1954

8. Merleau-Ponty, Maurice, Signes, Paris, nrf/Gallimard, 1960, p. 111. 
moderne, l'œuvre n'est pas expression mais création : elle donne à voir ce qui n'a pas été vu avant elle, elle forme au lieu de réfléter ${ }^{9}$ »

Le 6 novembre 1954, Repentigny définissait en ce sens la pratique des plasticiens, dans l'Autorité : il se référait d'ailleurs à la phénoménologie, en écrivant que "la démarche implicite dans cette peinture (celle des plasticiens) est du même ordre que celle qui caractérise la philosophie européenne contemporaine». Dans ce même article, il insiste aussi sur le verbe « constituer », qu'il oppose à « construire », faisant, là aussi, référence à la problématique phénoménologique capitale qu'est chez Husserl, par exemple, la constitution du sens par une conscience.

Ces traces de phénoménologie, nous les lisons aussi dans le Manifeste de 1955 où il nous est dit, au cinquième paragraphe, que les peintures plasticiennes procèdent par "réduction transcendantale ", autre opération éminemment phénoménologique.

Nous sommes donc chez Repentigny devant une conception de la peinture comme médium de découverte, conception qui va effectivement de pair avec celle que la phénoménologie se fait de l'art et de la philosophie elle-même ; dans sa préface à la Phénoménologie de la perception, Merleau-Ponty écrit encore :

"La philosophie n'est pas le reflet d'une vérité préalable, mais comme l'art la réalisation d'une véritét ${ }^{10}$."

C'est bien ce que veut dire Repentigny lorsqu'il écrit dans le Manifeste de 1955:

«Les Plasticiens n'admettent pas la postulation a priori de ce qui est élémentaire et de ce qui est parfait. Pour eux, ce ne sont pas là des données, mais des acquisitions que seul le travail individuel dans la plus entière liberté peut permettre de faire ${ }^{11}$.»

Il n'y a donc pas une vérité à dire par divers moyens

9. Picon, Gaétan, Liusage de la lecture, Paris, Mercure de France, 1960, tome II, p. 289.

10. Merieatj-Ponty, Maurice, Phénoménologie de la Perreption, Paris, nrf/Gallimard, 1945 , p. XV.

11. Manifeste des plasticiens, 10 février 1955. 
d'expression : c'est, au contraire, dans le creuset de l'expression qu'une vérité se constitue comme étant à dire. Pour Repentigny, dire une vérité préexistante conduit à utiliser des recettes techniques déjà élaborées, ce qui est la définition d'un art marqué par l'académisme et la répétition.

Un second principe qui caractérise une méthodologie de type herméneutique est l'inscription de l'objet à analyser, dans un contexte intersubjectif et psychologique.

La littérature ou la peinture deviennent alors une médiation, grâce à laquelle sont mis face ̀̀ face le «je»d'un spectateur et le « je » d'un sujet qui s'exprime. C'est l'auteur ou le peintre que l'on veut approcher et comprendre, à travers ses livres ou ses tableaux, la question étant celle de comprendre autrui dans et par les signes qu'il émet. L'on devine comment une telle méthode conduit à des études qui pourraient toutes porter en sous-titre «l'œuvre et l'homme», la valeur de l'œuvre trouvant son fondement dans un « je " exceptionnel.

Ici la position théorique de Repentigny est moins tranchée : disons qu'il est clair que c'est le pictural lui-même, et non l'homme, qui serait derrière le tableau, que sa critique cherche à comprendre et à expliquer. Il n'englobe pas l'espace pictural dans un espace intersubjectif où quelqu'un est à rencontrer, mais il considère le tableau comme un objet qui, bien que produit, n'en possède pas moins sa propre logique, cohérence et finalité à comprendre; par exemple le 10 juillet 1954, parlant de Toupin, Repentigny écrit :

"Il est parvenu à créer un espace pictural autonome et multiple, c'est l'espace d'un objet en soi ${ }^{12}$. "

Cependant lorsqu'il énonce directement quelques idées sur la relation production/tableau, il semble revenir davantage à l'idée d'un créateur génial qui soutient l'œuvre : il écrit, en décembre 1954, qu'un tableau de matière riche de formes pures et d'harmonies parfaites, "semblerait finalement la plus dérisoire des vanités s'il n’en sortait pas la chaleur de sa vérité humaine », et il conclut :

12. La Presse, "Des révélations au Petit Salon d'Été ", 10 juillet 1954. 
"En somme, on attend de l'artiste qu'il soit à la fois un saint et un héros. On l'acclame quand l'on sent qu'il nous donne l'exemple d'une richesse spirituelle intense ${ }^{13}$. »

Il conclut aussi qu'une "telle intensité d'être ne se retrouve guère que chez certains de nos peintres ${ }^{14}$ ".

De même, dans un article du 19 mars 1955, titré «Pas d'art vrai sans progrès intérieur ", il lie la vie intérieure de l'artiste à la valeur de sa production; cependant, au niveau de ce qu'il fait dans sa chronique d'art, Repentigny ne cesse de pratiquer une lecture de l'espace pictural qui est sans référence à un « je » et à ses aventures quotidiennes vitales. On n'y trouve guère de biographie et fort peu d'allusion aux drames personnels que vivraient les peintres. Avec Malraux, il affirme :

«Les peintres peignent des tableaux et non pas des êtres de la nature, non plus que des fantasmes de leur subjectivité $^{15}$."

Le principe herméneutique qui fait de l'œuvre la projection d'un « je», s'il est accepté donc dans quelques énoncés, nous semble néanmoins infirmé par l'ensemble de la pratique analytique qui est celle de Repentigny.

Le troisième principe de la critique herméneutique est une conséquence de ce contexte intersubjectif, dans lequel elle situe l'objet à interpréter : il affirme que l'objet artistique, littéraire ou pictural, est un « objet-sujet ", un " quasi-sujet », la forme extérieure de l'œuvre se dédouble en une forme intérieure de nature subjective, et c'est la tâche de l'interprète de dégager cette portée subjective; Repentigny ne semble guère tenir compte d'une telle dualité. Au contraire, il opère sans répit une réduction du sujet à l'intérieur du pictural.

Dans l'histoire de la peinture, telle que la comprend et l'énonce Repentigny, cette réduction du « je » est la dernière libération jusqu’à aujourd'hui.

En effet, un article contemporain du Manifeste, daté du 5

13. La Presse, "L'art enseigne l'habitude de la vérité ", 4 decembre 1954.

14. Ibidem.

15. La Presse, "Pas d'art vrai sans progrès intérieur ", 19 mars 1955 
février 1955 et intitulé «Un cycle dans l'histoire de l'art », trace les phases suivantes : jusqu'à la Renaissance, la peinture était soumise à un rituel sacré, d'ordre religieux. Mais lors de la Renaissance, elle s'affranchit de cette servitude pour adhérer à des valeurs humanistes et, par ce biais, devenir la servante d'un rituel «bourgeois » et "matérialiste "; au XIX e siècle, dit Repentigny, la peinture peu à peu s'affranchit de cette servitude «bourgeoise » pour devenir art libre, n'obéissant qu'à la sensibilité de l'artiste :

«C'est quand le peintre a voulu exprimer sans oripaux ses émotions et sa sensibilité, en peignant ce que son affectivité prenait comme objet, c'est alors qu'il a commencé à libérer l'art de la servitude à un idéal imposé de l'extérieur ${ }^{16}$. »

La domination du sujet dans l'espace peint, qui définit la «peinture poétique ", n’est vue que comme une étape, un intermédiaire vers une peinture totalement affranchie, et c'est Mondrian, comme l'affirme le Manifeste de 1955, qui « a permis de réduire l'ultime aliénation de l'œuvre peinte, soit l'extériorisation de la concentration sur soi-même ${ }^{18}$. »

Mondrian devient une référence parce qu'il a réussi « une élimination de toute mise en scène subjective »; «le peintre a voulu, écrivait en janvier 1955 Repentigny, à tout prix éviter de faire étalage de soi, de sa subjectivité19 ${ }^{19}$, au profit d'un équilibre pur obtenu par des moyens purement plastiques.

Ce n'est donc pas la cohérence d'une expérience subjective, ni une unité qui viendrait de la synthèse des expériences d'un même « moi », qui conferent une unité au tableau. Le tableau est un, uniquement à cause des éléments plastiques qui le composent et le constituent.

Ici Repentigny s'écarte donc de tout traitement herméneutique et avance nettement dans le sens d'une analyse structurale, intrinsèque et formelle. Le tableau n'est donc pas saisi comme un objet-sujet, comme une réalité ambiguë inclusive d'un «je » qui l'énonce et s'y inscrirait à la fois.

16. La Presse, "Quand même nous avons de vrais peintres", 27 mars 1954

17. La Patrie, "Un cycle dans l'histoire de l'art", 5 février 1955.

18. Manifeste des plasticiens, 10 février 1955.

19. La Presse, "Peinture et métamorphose sociale ", 22 janvier 1955. 
Quatrième trait de l'herméneutique: le champ des significations dégagées est un « champ de conscience "; il y a dans l'œuvre une intention, et c'est elle que l'on cherche à découvrir par l'interprétation.

Peut-être à cause des positions théoriques de l'automatisme et du surréalisme, Repentigny affirme aussi, à l'encontre de ce principe, les droits de l'inconscient en art : le Manifeste de 1955 renonce clairement à la peinture, conçue comme moyen d'expression conscient, et au contraire "n'exclut aucune des significations inconscientes possibles ". Aussi affirme-t-il qu'un tableau, à la limite, est sans cesse re-créé par la collectivité qui se le ré-approprie en lui donnant de nouvelles significations, peu importe qu'elles relèvent ou non des intentions conscientes de son auteur: on lit dans le Manifeste :

«Une auvre peut n'être pas la création de celui qui l'exécute, mais de celui qui la regarde ou d'une collectivité, plus simplement ${ }^{20}$."

Donc les significations d'un tableau ne sauraient être limitées par le champ de conscience intentionnel du « vouloir dire » d'un peintre.

Enfin, cinquième caractéristique d'un traitement herméneutique : comprendre, pour le spectateur, c'est reprendre à son compte, c'est revivre le sens et le réactiver : il faut une adhésion au tableau, on ne peut pas le comprendre en restant un spectateur-analyste étranger, on ne peut pas comprendre par l'analyse et la description seules.

Ici, Repentigny semble d'accord, et il demande aussi du spectateur une telle identification à l'œuvre peinte : il parle de " communion" nécessitant une "humilité " de la part du spectateur :

«C'est en cherchant à pénétrer le monde de l'expression plastique que l'on peut s'enrichir de visions nouvelles et d'intentions vitales. Cette pénétration n'est pas chose facile, toutefois, pour la plupart, nous en sommes empêchés par un " manque d'humilité ».. qui arrête les mouvements géné-

20. Manifeste des plasticiens, 10 février 1955. 
reux qui pourraient permettre des communions spontanées avec des belles créations de notre époque ${ }^{21}$."

Quelques jours plus tard, revenant sur ce thème, il affirme que l'art n'est servi que par une acceptation relevant d'une « réelle communion » ${ }^{22}$. En mars 1955 , il écrivait déjà que « toute cuvre de création demande une adhésion par l'intérieur ${ }^{23}$, et, l'année d'avant, que « le seul art valide est celui qui a l'adhésion de tout l'homme simultanément ${ }^{24}$.

Ce devoir du spectateur de permettre en quelque sorte que le tableau modifie réellement quelque chose dans sa vie, qu'il le convertisse pour ainsi dire, nous paraît conforme à l'idée que se fait l'herméneutique du geste qu'est comprendre une cuvre d'art.

Voilà donc rapidement cernés, en quelques points, les principes à partir desquels il nous semble que Repentigny analyse les productions plastiques: tout comme en art, il refusait, pour l'art national, d'utiliser des critères moins exigeants et un étalon inférieur à celui qui servait pour la scène internationale: en critique, son corpus théorique, avec ses hésitations, nous semble être au même diapason que la philosophie française d'après-guerre et faire siennes les principales affirmations de la plénoménologie naissante en France.

Pour terminer, je voudrais maintenant examiner quelques thèmes de la critique de Repentigny, qui permettront peutêtre de saisir en quoi, aujourd'hui, les problèmes qu'il pose se sont déplacés, ou, au contraire, sont bel et bien restés les mêmes : premièrement, Repentigny partage une conception de l'art, qui remonte à Platon et qui affirme que l'art a trait à la vérité. C'est bien de vérité pour lui qu'il s'agit en peinture :

"Dans une société qui n'assigne aucun rôle au peintre et au sculpteur, la fonction de ceux-ci devient, de fait, d'engendrer chez le spectateur une soif de vérité . . . L'œuvre d'art a toujours été, consciemment ou non, symbole de quelque chose. Maintenant, elle est symbole de la recherche angoissée de l'homme contemporain pour sa vérité quelle qu'elle soit ${ }^{25}$. ”

21. La Presse, "Pour nous apprendre à voir ", 14 mai 1955.

22. La Presse, "Malentendu sur la peinture contemporaine ", 19 mai 1955

23. La Presse, "Pas d'art vrai sans progrès intérieur ", 19 mars 1955.

24. La Presse, "L'art enseigne l'habitude de la vérité ", 4 décembre 1954.

25. La Presse, "L'œuvre doit avoir un destin vrai ", 24 décembre 1954. 
Le 4 décembre 1954, on lit que « le seul recours de l'artiste est de chercher de toute son énergie, à rendre par une œuvre, la vérité, sa vérité ${ }^{26}$.»

Le Manifeste, dans ses aphorismes, reprendra cette affirmation : «La création, y lit-on, qui est aussi intuition, est l'unique forme de la vérité » et « est respectable dans son intégrité tout art vrai ${ }^{27} »$.

Même si ce critère de vérité est difficile à définir, dans ses critiques Repentigny y revient souvent, notant par exemple en décembre 1954: "il demeure assez difficile de distinguer la vérité dans tout ce travail bien fait ${ }^{28}$. »

S'il est vrai, comme Marcuse croit le démontrer en analysant l'évolution même du concept d'esthétique, que l'art subit une nette répression en quittant le domaine de la sensualité et du principe de plaisir, pour se laisser définir par le principe de raison en termes de processus cognitifs, Repentigny est victime de cette idéologie en pensant l'art en liaison avec la vérité, et non avec le ludique et le plaisir. Ce n'est peut-être pas un hasard si, après le mouvement des premiers plasticiens, l'art au Québec s'est aussi dirigé vers des formes plus ludiques, comme la fête et le «happening ». La conception de l'art que diffuse Repentigny est à l'opposé de l'art comme amusement : Mondrian est encore ici l'exemple ; et son ceuvre, écrit Repentigny, est « la consommation de la révolte des peintres contre le métier d'amuseur ${ }^{29} \gg$.

Face à la dualité qui oppose l'art comme savoir à l'art comme jeu, Repentigny se range donc du côté du savoir, faisant de l'art une expérience de vérité et étant en cela fidèle au courant dominant de la civilisation.

Un second thème intéressant est celui des relations entre le non-figuratif géométrique et le matérialisme.

Si, aujourd'hui, certaines pratiques de la peinture non figurative se réclament du matérialisme et identifient la fin de

26. La Presse, «L'art enseigne l'habitude de la vérité ", 4 décembre 1954.

27. Manifeste des plasticiens, 10 février 1955

28. La Presse, "Figures, formes et graphismes ", 11 décembre 1954

29. La Presse. "Une exposition historique au Musée ", I juin 1955. 
l'espace illusionniste à l'affirmation de la matérialité du support et des pigments, cette adéquation entre surface et matérialisme ne se retrouve aucunement chez Repentigny, pour qui la pratique plasticienne de l'abstraction est une peinture plus spirituelle même qu'une toile classique à espace illusionniste. Il sent d'ailleurs à quelques reprises la nécessité de dissiper un malentendu qui justement accuse l'art abstrait d'être du matérialisme, reproche, dit-il, «erroné que l'on adresse trop souvent au mouvement contemporain en peintu$\mathrm{re}^{30} »$.

\section{Le 19 mai 1955, il écrit :}

«Dès qu'une auvre n'a pas un sujet qui conventionnellement appelle à la méditation, l'on entend des gens dire que c'est là une cuvre "bien dans la note du matérialisme contemporain », et qu'elle n'est que flatterie pour les sens . . . Ceux qui ne croient en la présence de l'esprit, que quand ils peuvent en lire les signes les plus convenus, les plus circonstanciés dans l'espace et le temps, les plus matériels en somme, ceux-là même auront la ridicule prétention de juger une ouvre d'art pour son contenu spirituel ${ }^{31}$. »

C'est justement, pense Repentigny, parce que la société est à tendance matérialiste, au sens où l'on ne s'intéresse qu'aux biens matériels, qu'une peinture spirituelle éprouve de la difficulté à se faire accepter.

"Il faudrait, écrit-il le 19 mars 1955, s'inquiéter si dans une société généralement admise comme "matérialiste", les arts plastiques s'incorporaient, tels qu'ils se présentent maintenant et sans obstacle, à la vie courante ${ }^{32}$."

Ce thème nous conduit directement à un autre thème majeur de Repentigny, celui des relations entre les productions artistiques et le monde ambiant, l'état de la civilisation où elles s'insèrent : examinons, pour conclure, ce thème fondamental chez lui.

Selon Repentigny, Mondrian et Kandinsky incarnent ici encore deux tendances :

30. La Presse, "Malentendu sur la peinture contemporaine ", 19 mai 1955.

31. lbidem.

32. La Presse, "Pas d'art vrai sans progrès intérieur ", 19 mars 1955. 
« La peinture de Mondrian a montré une voie aux artistes qui sentaient un besoin de s'insérer dans le cours de la civilisation pour la dépasser, la sublimer, tandis que celle de Kandinsky s'inscrit dans les rythmes de la nature et tend à y ramener l'homme ${ }^{33}$. »

L'alternative serait donc entre une peinture qui prend pour objet la nature, en extrait pour ainsi dire la rythmique essentielle et, en étant montrée, tend alors à ramener l'homme à la nature, et une autre peinture qui, elle, prend pour objet la civilisation, industrielle et urbaine, pour, comme l'écrit Repentigny, la dépasser et la sublimer, c'est-à-dire, là aussi, pour en produire un genre d'équivalent abstrait et spirituel qui parle à notre conscience.

Pour Repentigny, la peinture doit donc prendre, comme point de départ, l'environnement réel qui est celui de notre civilisation : en février 1956 et en mars 1957, il énonce ce choix :

« Notre vrai milieu vital, ce n'est plus la verdoyante nature, depuis longtemps, mais bien la scintillante et bruyante ville $\mathrm{e}^{34}$.

« Nous vivons dans une grande ville industrielle, au climat âpre, et nous voudrions que nos artistes nous offrent une porte de sortie, une évasion, une " oasis " où nous prélasser dans l'herbette! Ces artistes ne veulent plus nous amuser avec de gentils contes de fées $!^{35}$ ”

Il nous semble que ce choix est logique et qu'il est en corrélation avec le choix d'un are véridique, non d'un art ludique: Repentigny prône, non pas un art qui serait l'antidote du contexte social où nous vivons, non pas un art marginal où s'incarneraient le rêve et l'évasion, mais bien un art qui prend en charge son contexte et accepte de se confronter à lui.

Pour lui, comme il l'écrit le 11 février 1956, la peinture «a pour emploi d'établir un rapport entre les vivants et leur

33. La Presse, "Deux grands maitres de notre temps ", 31 juillet 1954.

34. La Presse, "L'art est aussi lien avec le monde ", 25 février 1956.

35. La Presse, "Quand la peinture se colore de l'âpreté de la ville industrielle ", 12 mars 1957. 
milieu, produit d'une industrie de plus en plus artificielle, c'est-à-dire humaine $^{36}$ », et, corrélativement, « le refus des formes nouvelles et dynamiques de l'art a son analogie dans le nostalgique refus des formes métropolitaines et industrielles de la $\mathrm{vie}^{37} \gg$.

Que l'acte de peindre prenne comme vis-à-vis les formes métropolitaines et industrielles ne signifie évidemment pas qu'il s'agit, après les paysages ruraux, de représenter maintenant des paysages urbains!

«Est-ce à dire, écrit Repentigny le 25 février 1956, que ces machines doivent de ce fait être les sujets des tableaux ? » Voilà qui serait quelque peu simpliste. «Le véritable « sujet» de l'œuvre d'un artiste n'est jamais que son rapport personnel au monde, qui n'a pas tellement besoin d'être pensé que d'être vécu $^{38}$. »

La tâche de la peinture est donc de visualiser plastiquement non pas les objets urbains eux-mêmes, mais le rapport vécu, la relation existentielle que nous entretenons avec eux, leur sens tel que nous en prenons conscience et le vivons.

Encore ici, cette relation de la peinture à son objet est d'ordre phénoménologique et constitue bien un exemple appliqué de ce qu'Husserl nomme l'E $\pi \circ \chi_{\eta} \eta$ ou la « réduction transcendantale", comme dira Jauran dans le Manifeste: il s'agit d'une opération qui met entre parenthèses le monde objectif, mais conserve le monde comme objet de ma pensée ; laissons Husserl lui-même s'expliquer :

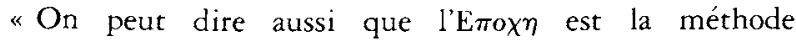
universelle et radicale par laquelle je me saisis comme moi pur, avec la vie de conscience pure qui m'est propre, vie dans et par laquelle le monde objectif tout entier existe pour moi . . . À vrai dire, le monde n'est pas pour moi autre chose que ce qui existe et vaut pour ma conscience, dans un pareil cogito . . . Je ne puis agir et porter des jugements de valeur dans un monde autre que celui qui se trouve en moi et tire de moi-même son sens et sa validité ${ }^{39}$.»

36. La Presse, "Toupin, Belzile et Bowles", 11 février 1956.

37. La Presse, "L'art est aussi lien avec le monde », 25 février 1956

38. Ibidem.

39. HuSSERL, Edmond, Méditations sartésiennes, Paris, Vrin, 1966, p. 18. 
C'est par le biais d'une telle démarche que la peinture plasticienne prétend "s'insérer dans le cours de la civilisation pour la dépasser et la sublimer ${ }^{40} "$; il s'agit de peindre non pas la ville existante, mais la ville dans l'expérience vécue que j'en ai, la ville en tant qu'expérience et objet de conscience, en partant du monde ambiant pour se l'annexer comme objet de conscience signifiant.

C'est d'ailleurs à ce niveau que Repentigny comprend la coupure qui met fin à l'attitude des automatistes et à leur révolte : il écrit, le 12 mars 1957, dans un article intitulé "Quand la peinture se colore à l'âpreté de la ville industrielle» :

"Si à une certaine époque - la belle époque de 1945 la plupart des peintres que nous connaissons maintenant comme non figuratifs, traversaient une phase de révolte émotionnelle contre les limitations imposées à l'expression par une vieille génération, il n'en va pas du tout de même aujourd'hui. Les Canadiens Français ont beau être considérés comme de gentils arriérés par pas mal de gens, rien n'oblige nos artistes à revêtir cet habit.

Actuellement (donc), plusieurs artistes prennent conscience des circonstances de leur existence - dans un monde où l'organisation est d'une complexité toujours croissante, et où instinct, habitudes et connaissances sont délégués à des machines aux prouesses illimitées. Il faut comprendre qu'il ne peut ici être question de révolte, la révolte était tout indiquée quand il s'agissait d'en finir avec les normes imposées par les représentants du passé : mais pour s'élever contre la machine, il faut avoir l'âme réactionnaire, ce dont nous n'avons nul besoin en ce moment. La seule attitude qui permette de survivre est celle de l'homme qui s'annexe la machine ${ }^{41}$. "

S'insérer dans notre civilisation, mais pas en la glorifiant comme telle, puisque, nous l'avons dit, elle est trop vulgairement matérialiste aux yeux de Repentigny; non pas en la posant dans sa réalité objective, car alors il y aurait nette contradiction : comment en effet, pourrait-on affirmer que cette peinture est «spirituelle», alors qu'elle est la résultante

40. La Presse, "Deux grands maitres de notre temps ", 31 juiller 1954.

41. La Presse, "Quand la peinture se colore à l'âpreté de la ville industrielle ", 12 mars 1957. 
et le double d'une société décrite comme matérialiste et qu'elle lui correspond?

Encore une fois, si Repentigny peut à la fois maintenir le caractère spirituel d'une peinture et sa correspondance à l'état de la civilisation matérialiste ambiante, c'est par le biais de la réduction transcendantale phénoménologique, qui fait que le tableau ne reproduit du monde que son sens immanent à la conscience, non sa réalité objective: "La meilleure façon d'humaniser ce monde technique, écrit-il en février 1956, est précisément de projeter à sa face les états d'esprit qu'il provoque en nous ${ }^{42}$."

C'est donc par cette réduction que l'art peut surmonter le dilemme suivant : accepter le monde ambiant, ou le fuir et le nier, dans un comportement passéiste et réactionnaire.

La réduction que j'ai rapidement décrite permet de dialectiser la relation art/vie collective : l'environnement dans lequel nous vivons a bel et bien subi des influences des artistes créateurs, mais à son tour cet environnement « joue dans les créations des artistes ${ }^{43}$ " et s'y retrouve : ce rapport vivant et dialectique est le meilleur gage d'un art vrai et le seul garde-fou contre toute forme d'académisme.

Du réel environnant au tableau, et du tableau au monde ambiant que l'on peut maintenant grâce aux artistes voir différemment : dans l'article titré "Pour nous apprendre à voir ", 14 mai 1955, nous lisons :

«Un des rôles majeurs du peintre et du sculpteur est de métamorphoser sans cesse notre ambiance, non pas physiquement mais spirituellement, en nous donnant demain une nouvelle vision de ce que nous avons regardé hier ... On sort du musée le plus souvent avec une détermination d'admirer ceci ou cela, sans voir le monde pour autant sous un jour nouveau. Et pourtant, c'est précisément cela qui est important, voir le monde à travers des créations poétiques et $\operatorname{rares}^{44}$."

Bouclons la boucle : cette tâche de la peinture, « nous

42. La Presse, "L'art est aussi lien avec le monde ", 25 février 1956

43. La Presse, "Le public, les peintres et leurs rapports ", 9 avril 1955.

44. La Presse, "Pour apprendre à voir ", 14 mai 1955. 
apprendre à voir le monde sous un jour nouveau », est celle-là même que Merleau-Ponty attribue à la phénoménologie en écrivant : "La vrai philosophie est de réapprendre à voir le monde, et en ce sens une histoire racontée »; ajoutons ici, avec Repentigny : «un tableau peint, peut signifier le monde avec autant de profondeur qu'un traité de philosophie ${ }^{45} »$. C'est là, le parti pris fondamental de Jauran-Repentigny.

Université du Québec

à Montréal

45. Meriend-Ponty, Maurice, Pbénaménologie de la perception, NRF/Gallimard, 1945. p. XVI. 